PROCEEDINGS OF THE

AMERICAN MATHEMATICAL SOCIETY

Volume 134, Number 5, Pages 1375-1381

S 0002-9939(05)08245-6

Article electronically published on November 17, 2005

\title{
COMBINATORICS OF RANK JUMPS IN SIMPLICIAL HYPERGEOMETRIC SYSTEMS
}

\author{
LAURA FELICIA MATUSEVICH AND EZRA MILLER
}

(Communicated by Michael Stillman)

\begin{abstract}
Let $A$ be an integer $d \times n$ matrix, and assume that the convex hull $\operatorname{conv}(A)$ of its columns is a simplex of dimension $d-1$ not containing the origin. It is known that the semigroup ring $\mathbb{C}[\mathbb{N} A]$ is Cohen-Macaulay if and only if the rank of the GKZ hypergeometric system $H_{A}(\beta)$ equals the normalized volume of $\operatorname{conv}(A)$ for all complex parameters $\beta \in \mathbb{C}^{d}$ (Saito, 2002). Our refinement here shows that $H_{A}(\beta)$ has rank strictly larger than the volume of $\operatorname{conv}(A)$ if and only if $\beta$ lies in the Zariski closure (in $\mathbb{C}^{d}$ ) of all $\mathbb{Z}^{d}$ graded degrees where the local cohomology $\bigoplus_{i<d} H_{\mathfrak{m}}^{i}(\mathbb{C}[\mathbb{N} A])$ is nonzero. We conjecture that the same statement holds even when $\operatorname{conv}(A)$ is not a simplex.
\end{abstract}

\section{INTRODUCTION}

Gelfand, Graev, Kapranov, and Zelevinsky GGZ87, GZK89 defined certain linear systems of partial differential equations, now known as A-hypergeometric or GKZ hypergeometric systems $H_{A}(\beta)$, whose solutions generalize the classical hypergeometric series. These holonomic systems are constructed from discrete input consisting of an integer $d \times n$ matrix $A$ along with continuous input consisting of a complex vector $\beta \in \mathbb{C}^{d}$. We assume throughout that the convex hull $\operatorname{conv}(A)$ of the columns of $A$ does not contain the origin. The matrix $A$ determines a semigroup ring $\mathbb{C}[\mathbb{N} A]$, and it was shown in GZK89 that the dimension $\operatorname{rank}\left(H_{A}(\beta)\right)$ of the space of analytic solutions of $H_{A}(\beta)$ is independent of $\beta$ whenever $\mathbb{C}[\mathbb{N} A]$ is CohenMacaulay.

Meanwhile, Adolphson showed that even when $\mathbb{C}[\mathbb{N} A]$ is not Cohen-Macaulay, the rank of $H_{A}(\beta)$ is independent of $\beta$, as long as $\beta$ is generic in a certain precise sense [Ado94. After Sturmfels and Takayama showed that the rank can actually go up for nongeneric parameters $\beta$ [ST98], Cattani, D'Andrea and Dickenstein CDD99 showed that if $\operatorname{conv}(\mathbb{N} A)$ is a segment (so $d=2$ ), then the rank does jump whenever $\mathbb{C}[\mathbb{N} A]$ fails to be Cohen-Macaulay. This result was generalized by Saito Sai02, who, using different methods, proved that there exist rank-jumping parameters for any non-Cohen-Macaulay simplicial semigroup $\mathbb{C}[\mathbb{N} A]$.

In this note, we use the combinatorics of $\mathbb{Z}^{d}$-graded local cohomology to characterize the set of parameters $\beta$ for which the rank goes up, in the simplicial case. Our premise, reviewed in Section 2, is the standard fact that a semigroup ring $\mathbb{C}[\mathbb{N} A]$

Received by the editors February 10, 2004 and, in revised form, December 3, 2004.

2000 Mathematics Subject Classification. Primary 33C70; Secondary 14M25, 13N10, 13D45, 52B20, 13C14, 16S36, 20M25.

(C)2005 American Mathematical Society Reverts to public domain 28 years from publication 
fails to be Cohen-Macaulay if and only if a local cohomology module $H_{\mathfrak{m}}^{i}(\mathbb{C}[\mathbb{N} A])$ is nonzero for some cohomological index $i$ strictly less than the dimension $d$ of $\mathbb{C}[\mathbb{N} A]$. After gathering some facts about $A$-hypergeometric systems in Section 3 , we prove in Theorem 11 the simplicial case of the following.

Conjecture. Assume that $\operatorname{conv}(A)$ has dimension $d-1$. The set of parameters $\beta \in \mathbb{C}^{d}$ such that $\operatorname{rank}\left(H_{A}(\beta)\right)$ is greater than the generic rank equals the Zariski closure $\left(\right.$ in $\left.\mathbb{C}^{d}\right)$ of the set of $\mathbb{Z}^{d}$-graded degrees where the local cohomology $\bigoplus_{i<d} H_{\mathfrak{m}}^{i}(\mathbb{C}[\mathbb{N} A])$ is nonzero.

Our original intent in writing this paper was to provide evidence for this conjecture by demonstrating in Theorem 11 its validity in the simplicial case. After this work was completed, the full conjecture was proved using a different approach [MMW04].

\section{Computing LOCAl COHOMOlogy For SEMigroup Rings}

Throughout this note, let $A$ be a $d \times n$ integer matrix whose first row has all entries equal to 1 , and whose columns $a_{1}, \ldots, a_{n}$ generate $\mathbb{Z}^{d}$ as a group. Unless otherwise explicitly stated, we do not assume that the polytope $\operatorname{conv}(A)$ obtained by taking the convex hull (in $\mathbb{R}^{d}$ ) of the column vectors $a_{1}, \ldots, a_{n}$ is a simplex; in particular, we need no simplicial assumptions from here through Definition 10, The semigroup

$$
\mathbb{N} A=\left\{\sum_{i=1}^{n} k_{i} a_{i} \mid k_{1}, \ldots, k_{n} \in \mathbb{N}\right\}
$$

has semigroup ring $R=\mathbb{C}[\mathbb{N} A] \cong \mathbb{C}\left[\partial_{1}, \ldots, \partial_{n}\right] / I_{A}$, where

$$
I_{A}=\left\langle\partial^{u}-\partial^{v} \mid A \cdot u=A \cdot v\right\rangle
$$

is the toric ideal of $A$. The ring $R$ is naturally graded by $\mathbb{Z}^{d}$, with the $i^{\text {th }}$ indeterminate having degree $\operatorname{deg}\left(\partial_{i}\right)=a_{i}$ equal to the $i^{\text {th }}$ column of $A$. By a face of $\mathbb{N} A$ we mean a set of lattice points minimizing some linear functional on $\mathbb{N} A$. The terms ray and facet refer to faces of dimension 1 and $d-1$, respectively, where the dimension of a face equals the rank of the subgroup $\mathbb{Z} \tau \subseteq \mathbb{Z}^{d}$ it generates. It is convenient to identify a face $\tau$ of $\mathbb{N} A$ with the subset of $\{1, \ldots, n\}$ indexing the vectors $a_{i}$ lying in $\tau$.

We now recall some facts from [BH93, Chapter 6] or [MS04, Chapter 13] about the local cohomology modules $H_{\mathfrak{m}}^{i}(R)$, where $\mathfrak{m}=\left\langle\partial_{1}, \ldots, \partial_{n}\right\rangle$ is the graded maximal ideal of $R$. Since $R$ is a semigroup ring, the local cohomology of $R$ is the cohomology of the complex

$$
0 \rightarrow R \rightarrow \bigoplus_{\text {rays } \tau} R_{\tau} \rightarrow \bigoplus_{\text {2-dim faces } \tau} R_{\tau} \rightarrow \cdots \rightarrow \bigoplus_{\text {facets } \tau} R_{\tau} \rightarrow R_{\mathfrak{m}} \rightarrow 0
$$

where $R_{\tau}$ is the localization of $R$ by inverting the indeterminates $\partial_{i}$ for $i \in \tau$. The differential is modeled on the algebraic cochain complex of the polytope $\operatorname{conv}(A)$, once orientations on the faces of $\operatorname{conv}(A)$ have been chosen.

The above local cohomology can be computed multidegree by multidegree. Indeed, the localization $R_{\tau}$ is nonzero in graded degree $\beta \in \mathbb{Z}^{d}$ if and only if $\beta$ lies in the subsemigroup $\mathbb{N} A+\mathbb{Z} \tau$ of $\mathbb{Z}^{d}$; that is, $R_{\tau}=\mathbb{C}[\mathbb{N} A+\mathbb{Z} \tau]$. Therefore, the faces 
of $\mathbb{N} A$ contributing a nonzero vector space (of dimension 1 ) to the degree $\beta$ piece of the complex (11) is

$$
\nabla(\beta)=\{\text { faces } \tau \text { of } \mathbb{N} A \mid \beta \in \mathbb{N} A+\mathbb{Z} \tau\} .
$$

This set of faces is closed under going up, meaning that if $\tau \subset \sigma$ and $\tau \in \nabla(\beta)$, then also $\sigma \in \nabla(\beta)$. When we write cohomology groups $H^{j}(\nabla)$ for such a polyhedral cocomplex, what we mean formally is that

$$
H^{j}(\nabla)=H^{j}(\operatorname{conv}(A), \operatorname{conv}(A) \backslash \nabla ; \mathbb{C})
$$

is the cohomology with complex coefficients of the relative cochain complex of the complementary polyhedral subcomplex of $\operatorname{conv}(A)$.

Here is a standard result in combinatorial commutative algebra.

Theorem 1. The local cohomology $H_{\mathfrak{m}}^{j}(R)_{\beta}$ of the semigroup ring $R$ in $\mathbb{Z}^{d}$-graded degree $\beta$ is isomorphic to $H^{j}(\nabla(\beta))$. In particular, $R$ is Cohen-Macaulay if and only if $H^{j}(\nabla(\beta))=0$ for all $\beta \in \mathbb{Z}^{d}$ and cohomological degrees $j=0, \ldots, d-1$.

Proof. Use the complex in (11) to compute local cohomology.

Definition 2 ([HM03). The sector partition is the partition of $\mathbb{Z}^{d}$ into equivalence classes for which $\beta \equiv \beta^{\prime}$ if and only if $\nabla(\beta)=\nabla\left(\beta^{\prime}\right)$. For a cocomplex $\nabla$, the (possibly empty) set of degrees $\beta \in \mathbb{Z}^{d}$ satisfying $\nabla(\beta)=\nabla$ is a sector.

Since the local cohomology of $R$ in degree $\beta$ only depends on $\nabla(\beta)$, it is constant on every sector.

Definition 3. A degree $\beta \in \mathbb{Z}^{d}$ such that $H^{j}(\nabla(\beta)) \neq 0$ for some $0 \leq j \leq d-1$ is called an exceptional degree of $A$. The Zariski closure of the set $E(A)$ of exceptional degrees of $A$ is called the slab arrangement $\bar{E}(A)$ of $A$. Given an irreducible component of the slab arrangement, the set of exceptional degrees lying inside that component and in no other components is called a slab.

Proposition 4. The slab arrangement is a union of affine translates of linear subspaces $\mathbb{C} \tau$ generated by faces $\tau$ of $\mathbb{N} A$, thought of as subsets of $\mathbb{C}^{d}$.

Proof. The Matlis dual of each local cohomology module is finitely generated, and therefore has a finite filtration whose successive quotients are $\mathbb{Z}^{d}$-graded shifts of quotients of $R$ by prime monomial ideals. Each successive quotient is therefore a $\mathbb{Z}^{d}$-graded shift of a semigroup ring $\mathbb{C}[\tau]$ for some face $\tau$ of $\mathbb{N} A$. The Matlis dual of local cohomology is thus supported on a set of degrees satisfying the conclusion of the proposition. The exceptional degrees are the negatives of the support degrees of the Matlis dual.

\section{Local COHOMOLOGY AND $A$-HyPeRgeOMEtRIC SYSTEMS}

Denote by $D_{n}$ the Weyl algebra, by which we mean the ring of linear partial differential operators with polynomial coefficients in $n$ variables. That is, $D_{n}$ is the free associative algebra $\mathbb{C}\left\langle x_{1}, \ldots, x_{n}, \partial_{1}, \ldots, \partial_{n}\right\rangle$ modulo the relations $x_{i} x_{j}-x_{j} x_{i}$, $\partial_{i} \partial_{j}-\partial_{j} \partial_{i}$ and $\partial_{j} x_{i}-x_{i} \partial_{j}-\delta_{i j}$, where $\delta_{i j}$ is the Kronecker delta.

Definition 5. Given $A=\left(a_{i j}\right)$ as before and $\beta \in \mathbb{C}^{d}$, the $A$-hypergeometric system with parameter $\beta$ is the left ideal in the Weyl algebra $D_{n}$ generated by

$$
I_{A} \quad \text { and } \quad \sum_{j=1}^{n} a_{i j} x_{j} \partial_{j}-\beta_{i} \quad \text { for } i=1, \ldots, d \text {. }
$$


The $A$-hypergeometric module with parameter $\beta$ is $M_{A}(\beta)=D_{n} / H_{A}(\beta)$.

The following result relates our way of computing local cohomology of semigroup rings to $A$-hypergeometric systems.

Theorem 6. Stratify $\mathbb{Z}^{d}$ so that $\beta$ lies in the same stratum as $\beta^{\prime}$ iff the D-modules $M_{A}(\beta)$ and $M_{A}\left(\beta^{\prime}\right)$ are isomorphic. This stratification refines the sector partition, meaning that $M_{A}(\beta) \cong M_{A}\left(\beta^{\prime}\right)$ implies $\nabla(\beta)=\nabla\left(\beta^{\prime}\right)$.

To prove this result, we recall Saito's combinatorial results on isomorphisms of hypergeometric $D$-modules.

Definition 7. Let $\beta \in \mathbb{C}^{d}$ and let $\tau$ be a face of the cone $\mathbb{N} A$. Let

$$
E_{\tau}(\beta)=\{\lambda \in \mathbb{C} \tau \mid \beta \in \lambda+\mathbb{N} A+\mathbb{Z} \tau\} / \mathbb{Z} \tau
$$

be the set of vectors $\lambda \in \mathbb{C} \tau$, up to translation by $\mathbb{Z} \tau$, for which $\beta-\lambda$ lies in the localization of $\mathbb{N} A$ along $\tau$.

Theorem 8 ([Sai02]). The D-modules $M_{A}(\beta)$ and $M_{A}\left(\beta^{\prime}\right)$ are isomorphic for two parameters $\beta$ and $\beta^{\prime}$ in $\mathbb{C}^{d}$ if and only if $E_{\tau}(\beta)=E_{\tau}\left(\beta^{\prime}\right)$ for all faces $\tau$ of $\mathbb{N} A$.

Proof of Theorem [6. Given a vector $\beta \in \mathbb{Z}^{d}$, we have

$$
\nabla(\beta)=\left\{\text { faces } \tau \text { of } \mathbb{N} A \mid 0 \in E_{\tau}(\beta)\right\}
$$

by definition. Therefore, for any pair of parameters $\beta, \beta^{\prime} \in \mathbb{Z}^{d}$ such that $M_{A}(\beta)$ is isomorphic to $M_{A}\left(\beta^{\prime}\right)$, we conclude that $\nabla(\beta)=\nabla\left(\beta^{\prime}\right)$ by Theorem 8 .

Remark 9. In general, the refinement in Theorem 6 is proper.

\section{RANK JUMPS IN THE SIMPLEX CASE}

Definition 10. A parameter vector $\beta \in \mathbb{C}^{d}$ is a rank-jumping parameter of $A$ if $\operatorname{rank}\left(H_{A}(\beta)\right)>\operatorname{vol}(A)$, where $\operatorname{vol}(A)$ is the normalized volume of the polytope $\operatorname{conv}(A)$. The set of rank-jumping parameters of $A$ is called the exceptional set of $A$, and denoted $\mathcal{E}(A)$.

Theorem 11. Fix a $d \times n$ integer matrix $A$. If $\operatorname{conv}(A)$ is a $(d-1)$-simplex, then the exceptional set $\mathcal{E}(A)$ is the Zariski closure $\bar{E}(A)$ of the set of exceptional degrees.

Remark 12. Computational evidence (using computer algebra systems Macaulay 2 GS, Singular GPS01, and $\mathrm{CoCoA}[\mathrm{CoC}]$ ) as well as heuristic arguments led us to conjecture the statement of Theorem 11. In fact, the evidence suggested that Theorem 11 generalizes to the case where $A$ is an arbitrary integer matrix. This has since been shown in a subsequent paper MMW04 via general geometric and homological methods.

Before getting to the proof, we need four preliminary results. The first two do not invoke the hypothesis that $\operatorname{conv}(A)$ is a simplex.

Lemma 13. Suppose that $\rho$ is a face of $\mathbb{N} A$, and $\alpha \in \rho$ is a vector not lying on any proper face of $\rho$. If $\beta \in \mathbb{Z}^{d}$, then the only localizations $\mathbb{N} A+\mathbb{Z} \mu$ capable of containing $\beta-m \alpha$ for all large (positive) integers $m$ are those for faces $\mu$ containing $\rho$. In other words,

$$
\mu \in \nabla(\beta-m \alpha) \text { for all } m \gg 0 \Rightarrow \mu \supseteq \rho \text {. }
$$


Proof. If $\mu$ does not contain $\rho$, then choose a linear functional that is zero along $\mu$ but positive on $\alpha$. This linear functional remains negative on $\beta-m \alpha+\gamma$ for all $m \gg 0$ and $\gamma \in \mu$, so that $\beta-m \alpha \notin \mathbb{N} A+\mathbb{Z} \mu$.

Lemma 14. Fix $\beta \in \mathbb{Z}^{d}$. Suppose that $\rho$ is maximal among faces of $\mathbb{N} A$ not in $\nabla(\beta)$, but that $\rho$ is neither $\mathbb{N} A$ nor a facet of $\mathbb{N} A$. If $\alpha \in \rho$ is a vector not lying on any proper face of $\rho$, then $\beta-m \alpha$ is an exceptional degree for all large integers $m$.

Proof. Suppose that $\mu$ contains $\rho$. Since $m \alpha \in \mathbb{Z} \mu$ for all integers $m$, we find that $\beta-m \alpha \in \mathbb{N} A+\mathbb{Z} \mu$ for all integers $m$ if and only if $\beta \in \mathbb{N} A+\mathbb{Z} \mu$. By Lemma 13 we conclude that $\nabla(\beta-m \alpha)$ is, for $m \gg 0$, the cocomplex of all faces strictly containing $\rho$. The cohomology of such a cocomplex is the same as that of a sphere having dimension $1+\operatorname{dim}(\rho)$ : a copy of $\mathbb{C}$ in dimension $1+\operatorname{dim}(\rho)$ and zero elsewhere. This cohomology is not in cohomological degree $d$ by the codimension hypothesis on $\rho$.

Lemma 15. Suppose that $\nabla$ is a cocomplex inside of a simplex of dimension e. If the cohomology $H^{j}(\nabla)$ is nonzero for some $j<e$, then there is a face $\xi$ of codimension at least 2 inside the simplex such that $\xi \notin \nabla$ but $\mu \in \nabla$ for all other faces $\mu$ containing $\xi$.

Proof. The equivalent dual statement is easier to visualize: If $\Delta$ is a simplicial complex inside of a simplex, and the reduced homology $\tilde{H}_{j}(\Delta)$ is nonzero in some homological degree $j \geq 0$, then there is a face $\xi$ of dimension at least 1 in the simplex such that $\xi \notin \Delta$ but $\mu \in \Delta$ for every proper face $\mu$ of $\xi$. Equivalently, $\Delta$ has a minimal nonface of dimension at least 1 . This statement reduces easily to the case where all vertices of the simplex lie in $\Delta$, and in that case one notes that every nonface has dimension at least 1 , assuming $\Delta$ has at least two vertices. But $\Delta$ has reduced homology in dimension $j \geq 0$, so it must have at least two vertices and at least one nonface.

Remark 16. Lemma 15 fails immediately for polyhedral cocomplexes that are not simplicial. Two parallel edges of a square (plus the interior cell of the square) form a polyhedral cocomplex that has cohomology of dimension 1 in cohomological degree 1, but the only two maximal nonfaces are the remaining two edges, of codimension 1.

Theorem 17 ([Sai02]). Suppose that the polytope $\operatorname{conv}(A)$ is a simplex. Then $\beta$ is a rank-jumping parameter of $A$ if and only if there exist faces $\sigma$ and $\tau$ of $\mathbb{N} A$, and an element $\lambda \in \mathbb{C} \sigma \cap \mathbb{C} \tau$, such that

$$
\lambda \in E_{\sigma}(\beta) \cap E_{\tau}(\beta) \text { but } \lambda \notin E_{\sigma \cap \tau}(\beta) .
$$

Proof of Theorem 11. We begin by showing that the exceptional set is contained in the slab arrangement. Let $\beta \in \mathbb{C}^{d}$ be a rank-jumping parameter of $A$, and pick $\sigma$, $\tau$ and $\lambda$ as in Theorem 17. For any $\alpha \in \mathbb{C} \sigma \cap \mathbb{C} \tau$, the sum $\beta+\alpha$ is a rank-jumping parameter, as can be seen by replacing $\lambda$ with $\lambda+\alpha$ in Theorem 17 and noting that

$$
\lambda+\alpha \in E_{\tau}(\beta+\alpha) \Longleftrightarrow \lambda \in E_{\tau}(\beta) .
$$

Therefore we may (and do) assume that $\beta \in \mathbb{Z}^{d}$ and $\lambda=0$.

Recall (2), which in particular implies that the set of faces $\mu$ satisfying $0 \in E_{\mu}(\beta)$ forms a cocomplex. This allows us to enlarge $\sigma$ and $\tau$ so that $\sigma \cap \tau$ is maximal 
among faces of $\mathbb{N} A$ outside $\nabla(\beta)$, while still satisfying Theorem 17. Taking $\rho=\sigma \cap \tau$ in Lemma 14, we find that $\beta-m \alpha$ is an exceptional degree for all $m \gg 0$ and all choices of $\alpha$ interior to $\rho$. The slab arrangement $\bar{E}(A)$ therefore contains $\beta+\mathbb{C} \rho$, and hence $\beta$.

Now suppose by Proposition 4 that $\beta+\mathbb{C} \rho$ is an irreducible component of the slab arrangement $\bar{E}(A)$, where $\beta \in \mathbb{Z}^{d}$ and $\rho$ is a face of $\mathbb{N} A$. We wish to show that $\beta+\mathbb{C} \rho$ consists of rank-jumping parameters. In fact, we shall produce $\sigma, \tau$, and $\lambda$ as in Theorem 17 satisfying $\rho=\sigma \cap \tau$, although we might harmlessly shift $\beta$ by some vector in $\mathbb{Z}^{d} \cap \mathbb{C} \rho$ first.

The component $\beta+\mathbb{C} \rho$ is the closure of some slab parallel to $\rho$, which must (perhaps after shifting $\beta$ by an element in $\mathbb{Z}^{d} \cap \mathbb{C} \rho$ ) contain $\beta-m \alpha$ for an integer point $\alpha$ interior to $\rho$ and all $m \gg 0$. Replace $\beta$ by $\beta-m \alpha$ for some fixed large choice of $m$. Lemma 13 implies that the cocomplex $\nabla(\beta)$ is contained in the simplex consisting of all faces of $\mathbb{N} A$ containing $\rho$. If $\rho$ has dimension $d-e-1$, then this simplex satisfies the hypotheses of Lemma 15. Therefore we can find a face $\xi$ containing $\rho$ and of dimension at most $d-2$, such that $\xi$ is a maximal nonface of $\nabla(\beta)$. Applying Lemma 14 to $\xi$ instead of $\rho$, we find that the component $\beta+\mathbb{C} \xi$ contains $\beta+\mathbb{C} \rho$, and still lies inside $\bar{E}(A)$. From this we conclude that $\rho=\xi$, because $\beta+\mathbb{C} \rho$ is an irreducible component of $\bar{E}(A)$.

In summary, given that $\beta+\mathbb{C} \rho$ is an irreducible component of the slab arrangement $\bar{E}(A)$, we have moved $\beta$ by an element in $\mathbb{Z}^{d} \cap \mathbb{C} \rho$ so that

$$
\rho \notin \nabla(\beta) \text {, but } \mu \in \nabla(\beta) \text { for all faces } \mu \text { strictly containing } \rho \text {. }
$$

Moreover, we have shown that $\operatorname{dim}(\rho) \leq d-2$. Therefore we can pick two faces $\sigma$ and $\tau$ strictly containing $\rho$ and satisfying $\rho=\sigma \cap \tau$. For each $\lambda \in \mathbb{C} \rho$, we find that $\beta+\lambda$ is a rank-jumping parameter by substituting $\lambda=0$ and $\alpha=\lambda$ in (3), then using (2), and finally applying Theorem 17 .

\section{ACKNOWLEDGMENTS}

The results in this note were obtained as part of a larger project joint with Uli Walther, to whom we are very grateful. We also thank Bernd Sturmfels for his encouragement throughout this project. The Mathematical Sciences Research Institute (MSRI) in Berkeley, California played a key role in the development of this project. In particular, the research reported in this article was completed while both authors were postdoctoral fellows at MSRI, where the first author was partially supported by a Postdoctoral Fellowship. The second author was supported by a National Science Foundation Postdoctoral Research Fellowship.

\section{REFERENCES}

[Ado94] Alan Adolphson, Hypergeometric functions and rings generated by monomials, Duke Math. J. 73 (1994), no. 2, 269-290. MR.1262208 (96c:33020)

[BH93] Winfried Bruns and Jürgen Herzog, Cohen-Macaulay rings, Cambridge University Press, Cambridge, 1993. MR 1251956 (95h:13020)

[CDD99] Eduardo Cattani, Carlos D'Andrea, and Alicia Dickenstein, The A-hypergeometric system associated with a monomial curve, Duke Math. J. 99 (1999), no. 2, 179-207. MR1708034 (2001f:33018)

[CoC] CoCoATeam, CoCoA: a system for doing computations in commutative algebra, available at http://cocoa.dima.unige.it/.

[GGZ87] I. M. Gelfand, M. I. Graev, and A. V. Zelevinsky, Holonomic systems of equations and series of hypergeometric type, Dokl. Akad. Nauk SSSR 295 (1987), no. 1, 14-19. 
[GPS01] G.-M. Greuel, G. Pfister, and H. Schönemann, Singular 2.0, A computer algebra system for polynomial computations, Centre for Computer Algebra, University of Kaiserslautern, 2001, http://www.singular.uni-kl.de/.

[GS] Daniel R. Grayson and Michael E. Stillman, Macaulay 2, a software system for research in algebraic geometry, available at http://www.math.uiuc.edu/Macaulay2/.

[GZK89] I. M. Gelfand, A. V. Zelevinsky, and M. M. Kapranov, Hypergeometric functions and toric varieties, Funktsional. Anal. i Prilozhen. 23 (1989), no. 2, 12-26. Erratum: Funktsional. Anal. i Prilozhen. 27 (1993), no. 4, 91. MR1011353 (90m:22025)

[HM03] David Helm and Ezra Miller, Algorithms for graded injective resolutions and local cohomology over semigroup rings, J. Symbolic Computation 39 (2004), 373-395. MR2168288

[MMW04] Laura Felicia Matusevich, Ezra Miller, and Uli Walther, Homological methods for hypergeometric families, J. Amer. Math. Soc. 18 (2004), no. 4, 919-941. MR2163866

[MS04] Ezra Miller and Bernd Sturmfels, Combinatorial Commutative Algebra, Graduate Texts in Mathematics, Springer-Verlag, New York, 2004. MR2110098

[Sai02] Mutsumi Saito, Logarithm-free A-hypergeometric series, Duke Math. J. 115 (2002), no. 1, 53-73. MR.1932325 (2004f:16041)

[ST98] Bernd Sturmfels and Nobuki Takayama, Gröbner bases and hypergeometric functions, Gröbner bases and applications (Linz, 1998), London Math. Soc. Lecture Note Ser., vol. 251, Cambridge Univ. Press, Cambridge, 1998, pp. 246-258. MR1708882 (2001c:33026)

Mathematical Sciences Research Institute, Berkeley, California 94720

Current address: Department of Mathematics, Texas A\&M University, College Station, Texas 77843

E-mail address: laura@math.tamu.edu

Mathematical Sciences Research Institute, Berkeley, California 94720

Current address: School of Mathematics, University of Minnesota, Minneapolis, Minnesota 56267

E-mail address: ezra@math.umn.edu 